\title{
Clinical Features and Treatment Response to Differentiate Idiopathic Peritonitis From Non- strangulating Intestinal Infarction Associated With Strongylus Vulgaris Infection in the Horse
}

Ylva Hedberg-Alm ( $\square$ ylva.hedberg.alm@uds.slu.se )

Swedish University of Agricultural Sciences

\section{Eva Tydén}

Swedish University of Agricultural Sciences

\section{Lena-Mari Tamminen}

Swedish University of Agricultural Sciences

Lisa Lindström

Swedish University of Agricultural Sciences

Karin Anlén

Evidensia Animal Hospital

Maria Svensson

University Animal Hospital, Swedish University of Agricultural Sciences

Miia Riihimäki

Swedish University of Agricultural Sciences

\section{Research Article}

Keywords: Equine, intestinal parasite, colic, rectal examination, prognosis

Posted Date: November 30th, 2021

DOI: https://doi.org/10.21203/rs.3.rs-964971/v1

License: (c) (i) This work is licensed under a Creative Commons Attribution 4.0 International License. Read Full License 


\section{Abstract}

Background: Peritonitis in horses secondary to non-strangulating infarction (NSII) has a grave prognosis, even after intestinal resection. In contrast, horses with idiopathic peritonitis respond well to medical treatment. Affected horses in both cases often show signs of both colic and systemic inflammation, but early diagnosis is crucial for optimal treatment and an accurate prognosis. One cause of NSII is thrombus formation secondary to Strongylus vulgaris larval migration. There has been a documented increase in $S$. vulgaris prevalence in Sweden since the implementation of selective anthelmintic treatment in 2007, which subsequently could result in a rise in NSII cases. In a retrospective clinical study, medical records from cases diagnosed with NSII or idiopathic peritonitis from three equine referral hospitals in Sweden during 2017-2020 were reviewed. Information including demographic data, relevant medical history, and clinical- and laboratory parameters were obtained from patient records. To facilitate the differentiation between cases of idiopathic peritonitis and cases with confirmed NSII, the aim of the study was to compare clinical and laboratory parameters, clinical progression and initial response to antimicrobial treatment as well as survival-rates.

Results: Horses with NSII $(n=20)$ were significantly more likely to present during the winter months with a poorer response to medical treatment within 48 hours. Cases of idiopathic peritonitis $(n=115)$ had a $99.1 \%$ survival rate with medical treatment. In comparison, all confirmed NSIl cases were non-responsive to antimicrobial treatment and had a low survival rate (25\%) with surgical treatment. Specific rectal findings and peripheral blood neutropenia were strongly associated with NSII.

Conclusions: In Sweden, idiopathic peritonitis cases still predominate over S. vulgaris associated NSII cases and have an excellent survival rate with antimicrobial treatment. However, horses presenting with septic peritonitis during the winter months with a palpable rectal mass and continuing fever and colic signs beyond 48 hours of medical treatment are likely to suffer from NSIl and should be considered for abdominal surgery.

\section{Background}

Acute primary or idiopathic peritonitis without an identifiable aetiology is reported to have very high survival rates $(94-100 \%)$ and although often described as uncommon, the disease is well recognized in some countries, such as Australia and Sweden. ${ }^{1,2,3}$ Peritonitis secondary to non- strangulating intestinal infarction (NSII), however, is associated with a grave prognosis with medical treatment alone and poor prognosis even after surgical resection of the affected intestinal segment (33\%). ${ }^{4}$ Although several other causes of NSIl have been suggested, ${ }^{5,6,7}$ infarctions of the intestinal wall secondary to mesenteric arterial thrombosis associated with Strongy/us vulgaris larval migration have been well described. $4,8,9$ Since the implementation of prescription-only anthelmintic treatment, and a more selective anthelmintic treatment strategy, Sweden and Denmark have documented a clear increase in $S$. vulgaris prevalence in the equine population. ${ }^{10,11}$ Recently, ELISA antibodies to $S$ vulgaris were shown to be present in $62 \%$ of horses, revealing a high level of exposure to the parasite in the Swedish horse population. ${ }^{12}$ 
Clinical cases of both NSII and idiopathic peritonitis often display varying degrees of colic accompanied by signs of systemic inflammation. ${ }^{3,4}$ In addition, both diseases present without signs of trauma or a history of potential risk factors, such as recent parturition or abdominal surgery. A study performed in Denmark suggested that, in areas where $S$. vulgaris is endemic, all horses admitted with septic peritonitis of an unidentifiable cause should undergo exploratory laparotomy. ${ }^{4}$ However, since idiopathic peritonitis is commonly identified in Swedish horses ${ }^{3}$, distinguishing between the two disease forms is important, both for optimal treatment and an accurate prognosis.

To enable early differentiation between cases of idiopathic peritonitis and cases with confirmed NSII, the aim of the present study was to compare clinical and laboratory parameters, clinical progression and initial response to antimicrobial treatment as well as survival-rates between horses diagnosed with idiopathic peritonitis and horses with confirmed NSII, using medical records from three Swedish equine referral hospitals during a four year period (2017-2020).

\section{Results}

\section{Included cases}

A total of 135 cases were included in the study and further divided into two groups: i) 115 cases were classified as idiopathic peritonitis cases without an identifiable cause of the peritonitis apparent during the duration of hospitalization, ii) 20 cases had confirmed NSII of the pelvic flexure, determined by abdominal surgery and pathological assessment of the resected intestine or full body necropsy.

\section{Excluded cases}

Fifteen cases were excluded from the main analyses, as they did not meet the set inclusion criteria for case selection. Of these, two cases had suspected parasitic lesions involving the jejunum. One case was suspected of NSIl at the pelvic flexure, but the resected intestine was not sent for pathological assessment, and one case had a small infarction in the colon that was not resected. The remaining 11 cases had rectal examinations and/or rectal ultrasonographic findings suggestive of a mass, thickened intestinal wall at the pelvic flexure, adhesions and/or suspected thrombus formation in the mesenteric vessels, without confirmative diagnosis. Although unconfirmed, it was acknowledged that these latter horses could represent NSII cases that responded to medical treatment and the progression of clinical signs and outcome in this group was therefore recorded. In addition, all 15 horses were included as a separate group (unconfirmed NSII cases) in the cluster analysis.

\section{Exploratory and descriptive analyses using cluster analyses}

In the cluster analyses (Figure 1) clinical diagnosis clustered closely to the parameters leucocyte count, neutrophil count, specific rectal findings (rectal mass/adhesions or thickened intestinal wall) as well as 
the outcome parameters euthanized and days at clinic (see box "a", Figure 1). The pattern suggested that confirmed NSII was associated with lower leucocyte and neutrophil counts. The majority of individuals with confirmed NSII showed high similarity and clustered together (see box "b", Figure 1.). The common aspects of the individuals in cluster "b" were that they presented primarily during the winter months with a rectal mass/adhesions or thickened intestinal wall and a poor outcome. Cluster "b" also included a subcluster (see box " $c$ ", Figure 1) with unconfirmed NSIl cases. In this group, about half of the horses survived to discharge and it appeared to include slightly older horses. The confirmed cases (cluster " $b$ ") also appeared to divide into subclusters based on clinical signs. Confirmed cases with more severe colic at home and at admission, with signs of endotoxaemia, and that were often taken to surgery clustered together (see box " $d$ ", Figure 1). Most of the remaining horses with unconfirmed or confirmed NSII were found in a larger cluster including horses diagnosed with idiopathic peritonitis (see box "e", Figure 1). The horses with unconfirmed NSII differed from the horses in cluster " $b$ " in that they presented earlier in the year (during autumn), survived to discharge and had less elevated total protein in the peritoneal fluid as well as less decreased leukocyte count in the blood (neutrophil counts were missing for the majority of these horses).

\section{Demographic data}

In the idiopathic peritonitis group, Warm-blooded horses (including Thoroughbreds/Arabians) were most common (54\%), followed by pony breeds (17\%), Icelandic horses (17\%), Standardbred trotters (6\%), and Cold bloods (5\%). One horse was of unknown breed. Similarly, Warmbloods predominated in the NSII group (72\%), with $17 \%$ Cold bloods, one Icelandic horse, one of pony breed and one Arabian. In two cases in the NSII group the breed was not recorded. The idiopathic case group consisted of $50 \%$ geldings, $42 \%$ mares and $8 \%$ stallions. Of the NSIl confirmed cases, $65 \%$ were geldings, and $30 \%$ mares, with one stallion. The mean $( \pm S D)$ and median age in the two groups were as follows: idiopathic $12.6( \pm$ $6.1)$ and 12 years; NSII $11.7( \pm 4.8)$ and 10.5 years. There were no significant differences in demographic data between groups (Suppl. Table 1a).

\section{Previous medical history and admission data}

No statistical differences in relevant medical history data was found (Suppl. Table 1b). Within both groups, the number of cases that had received anthelmintic treatment within six months of presentation was similar to the number not treated within this time-frame, although this data was missing in a large number of cases in the idiopathic case group (37\%). In total, seven cases with confirmed NSII lesions had been treated with an anthelmintic drug within six months of admission. Variables with a significant difference between the two study groups are presented in Table 1. There was a significant effect of season ( $p=0.001)$, with horses diagnosed with NSII more often presenting during the autumn/winter months (Table 1), which was, as shown in the cluster analysis (Figure 1), due to NSIl cases presenting primarily during the winter months (Dec-Feb). The predicted probability of a horse being diagnosed with NSII increased markedly over the winter months, compared to the summer season (Figure 2). Horses later 
confirmed with NSII more often presented with obvious colic signs requiring analgesics $(p=0.005)$, but without fever $(p=0.027)$ (Table 1). However, this was not clear from the cluster analysis, due to the few number of cases with grade 3 colic. By case definition, no palpable masses, thickened intestine or adhesions were recorded in the idiopathic cases. However, such rectal findings were present in $65 \%$ of horses confirmed with NSII lesions and showed close association with both confirmed and unconfirmed NSIl cases in the cluster analysis. A normal rectal examination was significantly more common in the idiopathic case group $(p<0.001)$, with only one case diagnosed with NSII having no abnormal rectal findings. Trans-abdominal ultrasound was performed in 18 horses in the idiopathic peritonitis group, showing an increase in the amount of peritoneal fluid in all horses and in one case, also a mildly thickened small intestinal wall. No rectal ultrasound examination was performed in this group. In the NSII group, four horses had trans-abdominal ultrasound examinations, seven horses had rectal ultrasound examinations, and one horse had both types of examinations. On the trans-abdominal ultrasound, findings included increased amount of peritoneal fluid $(n=2)$, thickened small intestine $(n=3)$ and thickened colon wall $(n=1)$. All rectal ultrasound examinations showed a thickened colon wall, a localized mass involving or adjacent to a thickened colonic wall and/or suspected adhesion formation. Rectal ultrasounds were only performed if there had been abnormal rectal findings suggestive of a mass, thickened colon wall or adhesion formation. Remaining clinical data obtained at admission did not differ between groups (Suppl. Table 1c).

Table 1. Variables with significant differences between study groups.

${ }^{a}$ Fischer's exact test, ${ }^{b}$ Kruskal-Wallis rank sum test, ${ }^{\dagger}$ for every increase of $1.0 \times 10^{9} / \mathrm{L},{ }^{\ddagger}$ for every increase in $5.0 \mathrm{~g} / \mathrm{L}$

\section{Laboratory parameters and parasitic diagnostics}

All blood samples included were obtained prior to surgical intervention. Haematocrit was excluded from the analysis since different haematological analysers were used rendering non-comparable values between cases. No horse in either study group presented with a haematocrit above $50 \%$. Elevated fibrinogen levels on first sampling occasion were significantly more common in the NSII group (Table 1, p $=0.012$ ), but cluster analyses showed idiopathic cases with similar results (Figure 1). Leukopenia, characterized by a neutropenia, was significantly more frequent in the NSII group compared to horses diagnosed with idiopathic peritonitis $(p<0.001$ ) (Suppl. Figure $1 a, 1 b)$ and cluster analyses showed close association with final diagnosis. The mean protein level in the peritoneal fluid was higher in horses diagnosed with NSII as compared to the idiopathic case group $(p=0.011)$. However, as seen in the cluster analysis, there were horses in the idiopathic group with comparable levels. No other laboratory parameters were different between the two study groups (Suppl. Table 1d).

\section{Antimicrobial treatment regimes}


Variable

Idiopathic, $n(\%) \quad N S I I, n(\%)$

$P$ - OR Cl

value

(95\%)

Season at presentation ${ }^{a}$

\begin{tabular}{lllllr} 
Spring/Summer & $44(38)$ & $2(10)$ & \multicolumn{3}{c}{ Reference variable } \\
\hline Autumn/Winter & $71(62)$ & $18(90)$ & 0.019 & 5.58 & $1.51-$ \\
& & & & & 36.16
\end{tabular}

Colic at admission ${ }^{a}$

No colic at admission

$35(30)$

$4(20)$

Reference variable

Colic grade at admission

\begin{tabular}{lccccc} 
Grade 1 & $70(61)$ & $12(60)$ & 0.509 & 1.50 & $\begin{array}{c}0.48- \\
5.67\end{array}$ \\
Grade 2 & $8(7)$ & $0(0)$ & 0.991 & N/A & N/A \\
\hline Grade 3 & $2(2)$ & $4(20)$ & 0.005 & 17.50 & $\begin{array}{l}2.62- \\
161.78\end{array}$ \\
\hline Fever at admission $(\geq 38.5)^{\mathrm{a}}$ & $54(93)$ & $4(20)$ & 0.027 & 0.27 & $\begin{array}{l}0.08- \\
0.80\end{array}$
\end{tabular}

Missing

$2 \quad 0$

Rectal examination ${ }^{a}$

\begin{tabular}{|c|c|c|c|c|c|}
\hline Mass/adhesions & $0(0)$ & $13(65)$ & $<001$ & $\begin{array}{l}\text { Part o } \\
\text { definit }\end{array}$ & $\begin{array}{l}\text { case } \\
n\end{array}$ \\
\hline Normal rectal exam & $54(51)$ & $1(5)$ & $<.001$ & 0.05 & $\begin{array}{l}0.00- \\
0.35\end{array}$ \\
\hline Missing & 8 & 0 & & & \\
\hline WBC (median [IQR] $)^{\mathrm{b}}$ & $\begin{array}{l}7.08[5.50 \\
9.62] \times 10^{9} / \mathrm{L}\end{array}$ & $\begin{array}{l}3.50[3.05 \\
5.01] \times 10^{9} / \mathrm{L}\end{array}$ & $<.001$ & $0.04^{+}$ & $\begin{array}{l}0.28- \\
0.64\end{array}$ \\
\hline Missing & 7 & 5 & & & \\
\hline Neutrophils (median [IQR]) & $\begin{array}{l}4.95[3.60 \\
7.22] \times 10^{9} / \mathrm{L}\end{array}$ & $\begin{array}{l}2.40[1.40 \\
3.19] \times 10^{9} / L\end{array}$ & $<.001$ & $0.55^{\dagger}$ & $\begin{array}{l}0.38- \\
0.75\end{array}$ \\
\hline Missing & 7 & 4 & & & \\
\hline Elevated fibrinogen level ${ }^{a}$ & $18(20)$ & $8(53)$ & 0.012 & 4.70 & $\begin{array}{l}1.28- \\
17.19\end{array}$ \\
\hline Missing & 23 & 5 & & & \\
\hline Peritoneal total protein level & $\begin{array}{l}40.00[34.00 \\
50.00] \mathrm{g} / \mathrm{L}\end{array}$ & $\begin{array}{l}50.00[40.00 \\
60.00] \mathrm{g} / \mathrm{L}\end{array}$ & 0.011 & $1.38^{\ddagger}$ & $\begin{array}{l}1.10- \\
1.78\end{array}$ \\
\hline
\end{tabular}


All but one case in the idiopathic peritonitis group received antibiotic treatment. The horse that was not treated with antibiotics had peritoneal fluid with only a mildly elevated nucleated cell count $\left(12,250 \times 10^{6}\right)$ and was discharged within five days from admission. The remaining 114 horses were treated with either intravenous penicillin $\mathrm{G}$ sodium (51 \%), penicillin $\mathrm{G}$ sodium and gentamicin (39\%), penicillin $\mathrm{G}$ sodium and trimethoprim sulphonamide $(5 \%)$ or various other combinations $(4 \%)$. There was a difference in initial treatment regimen between the three hospitals, with the majority of horses at hospitals one and three treated with penicillin G sodium only (76\% and $69 \%$, respectively), whereas the broad-spectrum antibiotic combination of penicillin $\mathrm{G}$ sodium and gentamicin dominated at hospital two (78\%). In the NSIl group, four of cases were treated with penicillin G sodium only, all at hospital one. Six horses were initially treated with intravenous penicillin $G$ sodium only, with the addition of either gentamicin $(n=5)$ or trimethoprim sulphonamide $(n=1)$ due to continuing fever $(n=4)$ or decision for surgery $(n=2)$. The remaining horses were treated with a combination of penicillin $\mathrm{G}$ sodium and gentamicin from admission.

\section{Progression of colic signs and fever during hospitalisation}

In the idiopathic case group, there was a successive decline in the percentage of cases with fever ( $\geq$ $38.5^{\circ} \mathrm{C}$ ) during the first 48 hours of antimicrobial treatment. In contrast, approximately one third of horses subsequently diagnosed with NSII were still febrile 48 hours after treatment initiation. However, in the NSII group, seven cases had surgical intervention and three cases were euthanized within this time-frame. Obvious signs of colic (grade 2 or 3 ) were uncommon in the idiopathic case group, with a further reduction in the number of cases showing colic over the first 48 hours of medical treatment. In contrast, the proportion of cases with colic in the NSII group increased over the first 48 hours of medical treatment, together with a progressive reduction in the number of horses on medical treatment due to surgery or euthanasia. For a summary of colic signs and fever during the first 48 hours of medical treatment, see Figure 3.

The majority of horses presenting with peritonitis and a palpable mass, adhesions or suspected thrombosis (confirmed and unconfirmed NSII cases) that were febrile or had obvious signs of colic 48 hours after initiated antimicrobial treatment, were either euthanized or taken to surgery (Figure 4). The remaining eight cases were treated medically and survived to discharge.

\section{Surgical findings}

One horse in the idiopathic case group was subjected to surgery due to a right dorsal displacement of the ascending colon without vascular compromise. In the confirmed NSIl group, all twelve cases where surgery was performed had marked changes involving a segmental portion of the pelvic flexure or the 
juncture of the dorsal colon and the pelvic flexure, with a clear demarcation between healthy and unhealthy bowel without signs of strangulation (Figure 7, panel A). The compromised area of colon measured from five to $40 \mathrm{~cm}$ in length. In eight cases, thickening of the mesentery and/or enlargement of mesenteric lymph nodes were described. Adhesion formation between the pelvic flexure and the cecum and abdominal wall was found in three cases, of which one had a focal perforating lesion. Thrombosis formation in a ventral mesenteric vessel was observed in one horse.

\section{Outcome}

All cases in the idiopathic peritonitis group were discharged after varying lengths of medical treatment, or medical treatment and surgical correction of a colon displacement in one case, with a survival to discharge rate of $100 \%$. Most horses were discharged within 6-15 days of admission (72\%). Thirty-two horses $(28 \%)$ were treated with antibiotics after discharge at home for a duration of two to eight days, although in $50 \%$ of cases treatment length at home was unknown.

In the NSIl group, twelve horses (60\%) had surgery performed between one to seven days after admission, with resection of the affected colon segment in ten cases. One horse was euthanized at surgery due to a ruptured bowel and another due to owner declination of colon resection. Five of the ten cases where colon resection had been performed survived to discharge, giving a short-time survival rate of $50 \%$ after colon resection. These cases had surgery performed at admission $(n=2)$, three $(n=1)$, four $(n=1)$ and seven days $(n=1)$ after presentation. One case that survived to discharge was subsequently euthanized nine months after surgery, due to stricture formation at the anastomosis site. Another five cases were euthanized between 4 to 25 days after colon resection due to persistent colic signs or persisting septic peritonitis. Two horses in addition developed wound infections, which, however, were not the reason for euthanasia. Eight cases, where surgery was declined by the owner based on the guarded prognosis, were euthanized one to four days from admission. A summary of the outcome in the NSII group is shown in Figure 5 . Horses with rectal findings suggestive of NSII had a survival rate to discharge of $38 \%$, as displayed in Figure 6.

\section{Pathology}

\section{Necropsy}

In the NSIl group, 9/20 (45\%) of the horses had the diagnosis confirmed at necropsy, with the remainder confirmed after pathological examination of the surgically resected portion of intestine. In all cases, as defined by the inclusion criteria, the intestinal lesions were localized in or near the pelvic flexure. In most necropsied cases (7/9), a well-defined segmental area of transmural necrosis with varying degrees of eosinophilic inflammation of the pelvic flexure was demonstrated, in one case resulting in a perforating intestinal lesion. In one horse, the necropsy was performed after resection, where the resected intestine had not been sent for histopathological assessment. However, necrosis and ulcerations were observed at 
the anastomosis site and the caudal and cranial mesenteric roots showed severe lesions with thrombosis and multiple eosinophilic granulomas compatible with injuries secondary to $S$ vulgaris larval migration. The segmental lesion in the final horse was described as a $40 \mathrm{~cm}$ long segmental haemorrhagic infarction with multiple ulcerations. The length of the affected portion of colon, when noted, was between 5 and $40 \mathrm{~cm}$. Endarteritis of the cranial or caudal mesenteric roots and/or major mesenteric branches was found in all of horses, with thrombus formation recorded in seven cases. In six cases, S. vulgaris larvae were found within the arterial and/or intestinal lesions (Figure 7, panel C). Reactive hyperplasia and haemorrhage of the mesenteric lymph nodes or eosinophilic lymphadenitis was observed in four of necropsied cases. In four cases, fibrinous to fibrous adhesions were found. In two of these horses, there was severe adhesion formation between the colon ascendens, the abdominal wall and colon descendens, involving the uterus in one case (Figure 7, panel B). An acute, subacute, purulent or fibrinopurulent peritonitis was recorded in all but one case, in which the necropsy was performed five days after resection of the affected portion of intestine.

One horse that underwent colon resection, with histopathological examination of the resected tissue, was subsequently sent for necropsy nine months later, after euthanasia, due to repeated colon impactions. A stricture formation was demonstrated at the anastomosis site, but there were no macroscopic lesions in the cranial or caudal mesenteric arteries or its branches. Histological evaluation of the mesenteric arteries was not performed.

\section{Resected intestine}

The remaining eleven cases in the NSII group, all underwent exploratory laparotomy with findings as described in the previous section. Colon resection was performed in all cases (one after euthanasia to confirm diagnosis) and histopathological evaluation of the resected intestine showed severe transmural lesions, with variable inflammatory, fibrinosuppurative to necrotic changes, in most cases with substantial eosinophilic inflammation. In nine cases, vascular lesions with thrombus formation were recorded. One of these cases was identified during surgery with thrombosis in a ventral mesenteric artery and in four cases, parasites consistent with $S$. vulgaris larvae were found.

\section{Discussion}

It has previously been concluded that, in countries where $S$. vulgaris is considered endemic, all horses presenting with septic peritonitis without traumatic origin should be subjected to surgery. ${ }^{4}$ In Sweden, however, as was shown in the present study, the vast majority of septic peritonitis cases are classified as idiopathic and respond to medical treatment with an excellent prognosis for survival. ${ }^{3}$ The challenge with a possible increase in the number of peritonitis cases secondary to NSII lies in early detection, in order not to subject horses to surgery unnecessarily, to make an accurate prognosis, and to avoid suffering in cases where medical treatment is most likely hopeless, and where surgery or euthanasia instead would be the only feasible options. Although the number of confirmed cases of NSII in the present study was low, the data suggest that horses with septic peritonitis presenting during the winter months and where 
there is a palpable thickening of the colon and/or unidentified mass are very likely to have a NSII secondary to $S$. vulgaris migration. A low blood neutrophil count, elevated fibrinogen levels and a high peritoneal protein level with persistent fever and colic signs beyond 48 hours of antimicrobial treatment should further alert the clinician that surgery may be indicated.

In accordance with a recent study by Odelros et al. (2019), idiopathic peritonitis cases were common and dominated at all three hospitals, with cases of confirmed NSII associated with vascular lesions caused by S. vulgaris larval migration less frequently diagnosed. ${ }^{3}$ Similarly, a recent study in the UK found NSII to be a rare occurrence, although these cases were deduced to be of a different aetiology to $S$. vulgaris larval migration. ${ }^{7}$ In Denmark, NSIl was diagnosed in a minority of referred colic cases, with an incidence of $2 \% .{ }^{4}$ However, in the latter study, the authors noted an apparent increase in incidence since the implementation of a more selective approach to anthelmintic and during the course of the study, horses presenting with peritonitis were increasingly taken to surgery at diagnosis, as clinicians became aware of the risk of NSII in this group of horses. In a country such as Sweden, where idiopathic peritonitis is common, this, however, could result in many cases taken to surgery unnecessarily. Nonetheless, in the present study, clinicians progressively recognized that horses presenting with both peritonitis and a palpable mass adjacent to the pelvic flexure were very likely to suffer from NSII and a decision for euthanasia in these horses, when the owners declined surgery, was taken early during hospitalization, within four days of admission.

In the present study, there was a clear discrepancy in month of presentation, with idiopathic cases admitted uniformly throughout the year, whereas cases with confirmed NSII or a palpable rectal mass suggestive of NSII primarily presented during the winter months. This difference could be due to the underlying aetiology, i.e. the prolonged migratory phase of $S$. vulgaris larvae over a period of 4-5 months from infection, ${ }^{13}$ and similar findings have been made in other studies. ${ }^{4,8}$ In contrast, NSII cases of other aetiologies presented evenly over the year. ${ }^{7}$

Interestingly, there were no differences between idiopathic and NSII cases in the proportion of horses that had received anthelmintic treatment within six months of admittance, with $35 \%$ of NSII cases reported to have been treated with an anthelmintic drug within this time. In a recent study, a similar percentage of horses were found to be positive on a faecal $S$. vulgaris PCR test, despite regular deworming at least annually or within six months of testing. ${ }^{12}$ Possible explanations for these findings include re-infection from infected pastures after treatment or a lower than expected effectiveness of anthelmintic drugs on the L5 stage. ${ }^{14,15}$

Septic peritonitis, regardless of underlying cause, is associated with a systemic inflammation reflected in elevated inflammatory markers in the blood. ${ }^{2,3,4,6,16}$ In the present study, the majority of horses had severely elevated SAA levels, with no differences detected between groups, supporting the common feature of acute systemic inflammation in both idiopathic peritonitis and NSII and in agreement with previous studies. ${ }^{3,4}$ In contrast, elevated fibrinogen levels at first sampling was more common in NSII 
horses. Fibrinogen increases more slowly than SAA in response to systemic inflammation, and the differences in fibrinogen level could reflect the duration of the disease process, with NSII cases presenting at a later stage. ${ }^{17}$

In earlier studies, horses with NSII caused by verminous arteritis were described to present with acute colic, followed by endotoxaemia and shock, ${ }^{18,19,20,21}$ similar to the description in a more recent casereport, where rupture of the ileum had occurred as a result of ischemic injury secondary to verminous arteritis. ${ }^{22}$ In the present study, two horses developed signs of acute severe circulatory compromise soon after admission, in one case secondary to rupture of the colon, but none of the remaining 18 cases showed such signs. This is in accordance with Pihl et al. (2018), where none of the 30 included NSII cases showed signs of shock at presentation and similar to the idiopathic case group in the present study. ${ }^{4}$ However, the total white blood cell count and neutrophil count differed between groups, with significantly lower neutrophil levels in the NSIl cases. Neutropenia is a common feature of endotoxaemia suggesting that, although often not obvious from the clinical examination, endotoxaemia was more often present in horses diagnosed with NSII. ${ }^{23}$

Although not a consistent feature, the infarctive lesion was palpable rectally in the majority of confirmed NSIl cases in the present study. All intestinal infarctions were, as dictated by inclusion criteria, located in the pelvic flexure, which is an area readily accessible during rectal palpation, and highlights the importance of always performing a rectal examination in horses presenting with peritonitis, regardless of colic signs. Similarly, Pihl et al. (2018) found that in one third of the cases there was a palpable mass consistent with the location of the infarced intestine and overall, $69 \%$ of the cases had an infarction located in the pelvic flexure. ${ }^{4}$ Considering a poor correlation between palpatory findings and pathology of the mesenteric root has been shown, careful palpation of the large intestine appears more useful in the diagnosis of NSII. ${ }^{5}$ Transabdominal ultrasound was not helpful in diagnosis, but rectal ultrasound was a useful diagnostic aid in cases where colon thickening and/or a palpable mass had been detected. Ultrasonography of the cranial mesenteric artery could perhaps also be considered, especially in cases where no palpable mass is present. ${ }^{24}$

During the first 48 hours after initiation of antimicrobial treatment, there was a successive reduction in the proportion of idiopathic cases with either fever or obvious colic signs. In contrast, the proportion of horses with colic increased over the first 48 hours in the NSII group, with fever still present in about one third of cases. However, since necropsy or pathological assessment of intestinal tissue was required for confirmation of NSII, only horses that subsequently were taken to surgery or were euthanized could be included in the NSII group. Thus, a major weakness in the study is that horses with NSIl lesions that responded to medical treatment could have been missed. When considering all horses in the present study with rectal or ultrasonographic findings suggestive of NSII, regardless of confirmation, the overall short term survival rate was $37.5 \%$, which is considerably lower than that of the idiopathic peritonitis case group (100\%). 
Eight cases with rectal or ultrasonographic findings suggestive of NSIl survived with medical treatment alone and could represent horses in the early stages of NSIl with subsequent healing of the infarced area,

as has been suggested previously. 4,25 Whether some of the horses in the idiopathic case group also had smaller intestinal infarctions not palpable rectally and responding to medical treatment remains unverified. Parasitic migration of $S$. vulgaris with translocation of bacteria across the intestinal wall has been suggested as a possible cause of peritonitis in idiopathic cases, ${ }^{1,2,26}$ based on the isolation of Actinobacillus spp. from verminous aneurisms in the cranial mesenteric artery, ${ }^{27}$ highlighting the complexity in case differentiation. Recently, analysing serum for a $S$. vulgaris ELISA test, a significantly higher score was found in horses diagnosed with peritonitis compared to controls, even though the majority of cases were termed idiopathic. ${ }^{12}$

\section{Conclusion}

The results of the study supports medical treatment and a cautious approach to surgical decision in cases of peritonitis in countries such as Sweden where idiopathic peritonitis cases predominate. However, until specific markers for diagnosing $S$. vulgaris larval migration become available, clinicians working in countries where the parasite is endemic, should still be aware that horses presenting with septic peritonitis, particularly during the winter months, could suffer from intestinal infarction secondary to larval migration. In order to identify potential surgical cases, horses with septic peritonitis should be carefully evaluated, with particular emphasis on findings of rectal palpation and initial response to antimicrobial treatment.

\section{Methods}

The study was conducted as a retrospective clinical study using patient records from three Swedish equine referral hospitals during the years 2017-2020.

\section{Case selection}

For inclusion of idiopathic peritonitis cases, all cases with a diagnosis of peritonitis made within 48 hours of admission were reviewed. Overall inclusion criteria were a peritoneal fluid cell count $>10,000 \times 10^{6}$ in horses where no identifiable cause for the peritonitis was found during hospitalization. Horses with palpable masses, thickening of the intestinal wall or suspected thromboembolism, either on rectal or ultrasound examination, were excluded.

Regarding the NSII group, only horses with a confirmed diagnosis at necropsy or during explorative laparotomy were included. Criteria included a well demarcated ischemic lesion in close proximity of the pelvic flexure, which is an area with extensive collateral circulation and therefore prone to ischemia caused by arterial thromboembolism in the intestine. Horses had no signs of volvulus, other strangulating intestinal lesions or incarceration. Signs of verminous arteritis of the cranial or caudal mesenteric roots 
and its branches, as well as the presence of $S$. vulgaris larvae, were recorded if present, but not regarded vital for inclusion, since such lesion are difficult to ascertain without necropsy.

\section{Medical records review}

Information obtained from the medical records is summarised in Table 2. In brief, information included demographic data, immediate history and clinical parameters at admission. Laboratory parameters included blood analyses, peritoneal fluid analyses and parasitic diagnostics. Hospital data regarding antimicrobial treatment regimen, outcome (non-survivour/days until discharge), surgical, pathological and necropsy reports were also used. Colic signs were graded as follows: grade 0 : no colic; grade 1: decreased appetite/dull demeanour; grade 2: obvious colic signs (restlessness and pawing at the ground, irritated kicking to the stomach, rolling or attempting to roll); grade 3: obvious colic signs as grade 2 and requiring analgesics. Only grades 0-2 were used for grading of colic signs at home and grades 0-3 during hospitalization.

Table 2

Summary of data obtained from the medical records.

\begin{tabular}{|c|c|}
\hline Parameter & Description \\
\hline $\begin{array}{l}\text { Demographic } \\
\text { data }\end{array}$ & Age, sex, breed \\
\hline $\begin{array}{l}\text { Immediate } \\
\text { medical } \\
\text { history }\end{array}$ & $\begin{array}{l}\text { Colic signs }{ }^{\dagger}, \text { rectal temperature, medical treatment }\left(\text { NSAID }^{a} \text {, antibiotics), }\right. \\
\text { anthelmintic treatment past six months }\end{array}$ \\
\hline $\begin{array}{l}\text { Admission } \\
\text { data }\end{array}$ & $\begin{array}{l}\text { Month of presentation, heart rate, respiratory rate, mucous membranes (colour, crt }{ }^{\mathrm{b}} \text { ), } \\
\text { colic signs } s^{\ddagger} \text {, rectal temperature, rectal examination, abdominal/rectal ultrasound } \\
\text { examination }\end{array}$ \\
\hline \multirow[t]{3}{*}{$\begin{array}{l}\text { Laboratory } \\
\text { data }\end{array}$} & Blood: $W^{2} C^{c}$, neutrophil count, $\mathrm{PCV}^{\mathrm{d}}, \mathrm{TP}^{\mathrm{e}}, \mathrm{SA}^{\mathrm{f}}$, fibrinogen \\
\hline & Peritoneal fluid: $\mathrm{WBC}^{\mathrm{c}}, \mathrm{TP}^{\mathrm{e}}$, bacterial culture \\
\hline & Fecal sample: FEC ${ }^{g}$, S. vulgaris (PCR/culture), A. perfoliata \\
\hline Hospital data & $\begin{array}{l}\text { Colic signs }{ }^{\ddagger} \text {, rectal temperature, antimicrobial treatment regime, days of } \\
\text { hospitalization, outcome (non-survivor/days until discharge), surgical-, pathology- } \\
\text { and necropsy reports }\end{array}$ \\
\hline
\end{tabular}

${ }^{\dagger}$ Graded as 0: no colic signs, grade 1: dull demeanour/anorexia, grade 2: obvious colic signs

‡ Graded as 0: no colic signs, grade 1: dull demeanour/anorexia, grade 2: obvious colic signs, grade 3 : obvious colic signs requiring analgesics

${ }^{a}$ non-steroidal anti-inflammatory drug; ${ }^{b}$ capillary refill time; ${ }^{c}$ white blood cell count; ${ }^{d}$ packed cell volume; ${ }^{e}$ total protein; ${ }^{f}$ serum amyloid $A ;{ }^{g}$ faecal egg count. 


\section{Laboratory Analyses}

Analytical method used was primarily hospital dependent, but also dependent on the time of day a case was admitted (i.e. working hours or out-of-hours). Blood analyses for WBC and differential was performed by either Advia 2021i (Siemens, Erlangen, Germany), Procyte Dx (IDEXX, Hoofddorp, Netherlands) or Exigo H400 (Boule, Spånga, Sweden). Analyses for SAA was performed either by Architect C4000 (Abbot Core Laboratory, Abbott Park, Illinois, U.S.A.), Konelab 30i (Thermo Fisher Scientific, Waltham, U.S.A.) or StableLab (Epona Biotech Ltd, Sligo, Ireland) and fibrinogen by either Architect C4000 (Abbot Core Laboratory, Abbott Park, Illinois, U.S.A.) or Konilab 30i (Thermo Fisher Scientific, Waltham, U.S.A.). Peritoneal analyses for WBC was performed by either Advia 2021i (Siemens, Erlangen, Germany), Procyte Dx (IDEXX, Hoffddorp, Netherlands), Exigo H400 (Boule, Spånga, Sweden), Hemocue ${ }^{\circledR}$ WBC system (Radiometer, Crawley, England) or by manual count using a Bürker chamber. Peritoneal total protein was analysed using a refractometer (AO veterinary refractometer, U.S.A.).

\section{Parasite Diagnostics}

Strongyle faecal egg counts (FECs) were carried out for each horse using a modified McMaster technique with a minimum detection limit of $50 \mathrm{EPG} .^{28} \mathrm{~S}$. vulgaris diagnostic method used was hospital dependent by either Larval cultures on $50 \mathrm{~g}$ faeces according to Bellaw and Nielsen ${ }^{29}$ and examined under the microscope using morphological criteria ${ }^{30}$ or $S$. vulgaris specific $\mathrm{PCR}^{11,31}$. Anoplocephala perfoliata were examined on $30 \mathrm{~g}$ faeces using the modified flotation technique described by Berozoa et al. ${ }^{32}$

\section{Statistical analyses}

Excel was used for entering data and for descriptive analysis. Statistical analysis to study characteristics and potential indicators of horses with NSII was performed in R Statistical Software. ${ }^{33}$ A cluster analysis including clinic, diagnosis, outcomes, horse characteristics and clinical information available upon admittance was performed using the CluMix package. ${ }^{34,35}$ Variables were clustered by distance correlation. In addition, a descriptive and univariable analysis comparing horses with confirmed NSII and idiopathic peritonitis was performed. Quantitative measures were analysed using Kruskal-Wallis Rank Sum Test (non-parametric method) and qualitative variables using Fisher exact test (due to small number of observations) using the package tableone. ${ }^{36}$ Odds ratios and $95 \%$ were calculated using univariable logistic regression using the packages MASS and oddsratio. Seasonal effects of admittance of NSII patients was explored using a generalised additive model with a cyclic spline and clinic included as a random effect. ${ }^{37}$

\section{Abbreviations}

NSII: non-strangulating intestinal infarction

NSAID: non-steroidal anti-inflammatory drug 
SAA: serum amyloid $A$

FEC: faecal egg count

Epg: eggs per gram

Crt: capillary refill time

WBC: white blood cell count

PCV: packed cell volume

TP: total protein

\section{Declarations}

\section{Ethics approval and consent to participate}

Not applicable.

\section{Consent for publication}

Not applicable, retrospective study showing data from unidentifiable patients.

\section{Availability of data and materials}

The dataset used and analysed during the current study is included as a supplementary file ("Data set all horses") and can also be requested from the corresponding author: ylva.hedberg.alm@uds.slu.se.

\section{Competing interests}

The authors declare that they have no competing interests.

\section{Funding}

The study was funded by the Swedish-Norwegian foundation for Equine Research. The funding body did not influence the design, analysis or reporting of the study.

\section{Authors' contributions}

Y.H.A. provided clinical interpretation, conceived and devised the analyses, acquisitioned and interpreted the data for the article, drafted and revised the article. E.T. provided main scientific input in the field of equine parasitology, revised the article, wrote parasite diagnostics section, contributed to figure design. L.M.T. performed and interpreted the statistical analysis. 
L. L. interpreted the pathology and necropsy reports and revised the article in particular relating to description of pathological findings. K. A. provided clinical interpretation, acquisitioned data, revised the article. M. S. acquisitioned data. M.R. provided clinical interpretation, conceived and devised the analyses, acquisitioned and interpreted data for the article, revised the article.

\section{Acknowledgements}

We wish to acknowledge the Specialist Equine Hospital, Evidensia, Helsingborg, Equine Clinic, the University Animal Hospital, Swedish University of Agricultural Sciences, and Mälaren Equine Practice, Sigtuna, for providing access to medical records, which was greatly appreciated.

\section{References}

1. Golland LC, Hodgson DR, Hodgson JL. Peritonitis associated with Actinobacillus equuli in horses: 15 cases (1982-1992). J Am Vet Med Assoc. 1994; 205:340-3.

2. Matthews S, Dart AJ, Dowling BA, et al. Peritonitis associated with Actinobacillus equuli in horses: 51 cases. Aust Vet J. 2001; 79:536-9. doi: 10.1111/j.1751-0813.2001.tb10741.x.

3. Odelros $E$, Kendall A, Hedberg-Alm Y, et al. Idiopathic peritonitis in horses: a retrospective study of 130 cases in Sweden (2002-2017). Acta Vet Scand. 2019; 61:18. doi: 10.1186/s13028-019-0456-2.

4. Pihl TH, Nielsen MK, Olsen SN, et al. Nonstrangulating intestinal infarctions associated with Strongylus vulgaris: Clinical presentation and treatment outcomes of 30 horses (2008-2016). Equine Vet J. 2018; 50:474-480. doi: 10.1111/evj.12779.

5. N A White 2nd. Intestinal infarction associated with mesenteric vascular thrombotic disease in the horse. J Am Vet Med Assoc. 1981; 178:259-62.

6. Mair TS, Pearson GR. Multifocal non-strangulating intestinal infarction associated with larval cyathostomiasis in a pony. Equine Vet J. 1995; 27:154-5. doi: 10.1111/j.2042-3306.1995.tb03054.x.

7. Spanton JA, Mair TS, Sherlock CE, et al. Non-strangulating intestinal infarction in horses in the UK: A review of 15 cases. Equine Vet Educ. 2019; 32: 603-610. doi.org/10.1111/eve.13129.

8. Vibe-Petersen G, Nielsen K. Verminous enteritis and thrombo-embolic colic in the horse. A description of 36 cases (author's transl). Nord Vet Med. 1979; 31:385-91.

9. Nielsen MK, Jacobsen S, Olsen SN, et al. Nonstrangulating intestinal infarction associated with Strongylus vulgaris in referred Danish equine cases. Equine Vet J. 2016; 48:376-9. doi: 10.1111/evj.12422.

10. Nielsen MK, Vidyashankar AN, Olsen SN, Monrad J, et al. Strongylus vulgaris associated with usage of selective therapy on Danish horse farms-is it re-emerging? Vet Parasitol. 2012;189:260-6. doi: 10.1016/j.vetpar.2012.04.039.

11. Tydén E, Larsen Enemark $H$, Andersson Franko M, et al. Prevalence of Strongylus vulgaris in horses after ten years of prescription usage of anthelmintics in Sweden. Vet Parasitol X. 2019;2:100013. doi: 10.1016/j.vpoa.2019.100013. 
12. Hedberg-Alm, Y, Penell J, Riihimäki M, et al. Parasite Occurrence and Parasite Management in Swedish Horses Presenting with Gastrointestinal Disease-A Case-Control Study. Animals (Basel). 2020; 10:638. doi: 10.3390/ani10040638.

13. McCraw BM, Slocombe JO. Strongy/us vulgaris in the horse: a review. Can Vet J. 1976; 17:150-7.

14. Borji H., Moosavi Z., Ahmadi F. Cranial mesenteric arterial obstruction due to Strongylus vulgaris larvae in a Donkey (Equus asinus) Iran. J. Parasitol. 2014; 9:441-444.

15. Nielsen M.K., Scare J., Gravatte H.S., Bellaw J.L., Prado J.C., Reinemeyer C.R. Changes in Serum Strongylus vulgaris-Specific Antibody Concentrations in Response to Anthelmintic Treatment of Experimentally Infected Foals. Front. Vet. Sci. 2015; 2:17. doi: 10.3389/fvets.2015.00017.

16. Stewart AJ. Actinobacillus pleuritis and peritonitis in a quarter horse mare. Vet Clin North Am Equine Pract. 2006; 22: 77-93. doi: 10.1016/j.cveq.2005.12.023.

17. Jacobsen, S. Review of Equine Acute-Phase Proteins. AAEP Proceedings, Vol 53, 2007.

18. Enigk K. Zur Entwicklung von Strongylus vulgaris (Nematoden) im Wirtstier. Z. Tropenmed. Parasitol. 1950; 2:287-306.

19. Drudge JH, Lyons ET, Szanto J. Pathogenesis of migrating stages of helmimths, with special reference to Strongylus vulgaris. In: Soulsby EJL: Biology of parasites. Emphasis on veterinary parasites. New York and London: Academic Press Inc.; 1966: 199-214.

20. Duncan JL, Pirie HM. The pathogenesis of single experimental infections with Strongylus vulgaris in foals. Res. Vet. Sci. 1975; 18: 82- 93.

21. Drudge JH. Clinical aspects of Strongylus vulgaris infection in the horse. Vet. Clin. N. Am. - Large. 1979; 1:251-265.

22. Fjordbakk T, Gunnes, G. Circumferential lleal Rupture due to Verminous Arteritis in a Pony: A Case Report. J Eq Vet Sci. 2012; 32:638-640. doi.org/10.1016/j.jevs.2012.02.010.

23. Lilliehöök I, Tvedten HW, Bröjer J, et al. Time-related changes in equine neutrophils after experimental endotoxemia: myeloperoxidase staining, size, and numbers. Vet Clin Pathol. 2016; 45:66-72. doi: 10.1111/vcp.12334.

24. Wallace KD, Selcer BA, Tyler DE, et al. Transrectal ultrasonography of the cranial mesenteric artery of the horse. Am J Vet Res. 1989; 50:1699-1703.

25. Mair TS, Hillyer MH, Taylor FG. Peritonitis in adult horses: a review of 21 cases. Vet Rec. 1990; 126:567-70.

26. Rycroft AN, Garside LH. Actinobacillus species and their role in animal disease. Vet J. 2000; 159:1836. doi: $10.1053 /$ tvjl.1999.0403.

27. Dimock WW, Edwards PR, Bruner DW. Infections observed in equine fetuses and foals. Cornell Vet. 1947; 37:89-99.

28. Coles GC, Bauer C, Borgsteede FH, et al. World Association for the Advancement of Veterinary Parasitology (W.A.A.V.P.) methods for the detection of anthelmintic resistance in nematodes of veterinary importance. Vet. Parasitol. 1992; 44:35-44. 
29. Bellaw JL, Nielsen MK. Evaluation of Baermann apparatus sedimentation time on recovery of Strongylus vulgaris and Strongylus edentatus third stage larvae from equine coprocultures. Vet. Parasitol. 2015; 211:99-101. doi: 10.1016/j.vetpar.2015.05.001.

30. Russell AF. The development of helminthiasis in thoroughbred foals. J. Comp. Pathol. Therap. 1948; 58: 107-127.

31. Nielsen, M.K., Peterson, D.S., Monrad, J., Thamsborg, S.M., Olsen, S.N., Kaplan, R.M.,

32. Detection and semi-quantification of Strongylus vulgaris DNA in equine faeces by real-time quantitative PCR. Int. J. Parasitol. 38, 443-453. doi: 10.1016/j.jpara.2007.07.014.

33. Beroza GA, Marcus LC, Williams R, et al. Laboratory diagnosis of Anoplocephala perfoliata infection in horses. Proc. Am. Assoc. Equine Pract. 1987; 32:435-439.

34. R Core Team. R: A language and environment for statistical computing. R. Foundation for Statistical Computing, Vienna, Austria. 2020. URL https://www.R-project.org/.

35. Hummel M, Edelmann D, Kopp-Schneider A. Clustering of samples and variables with mixed-type data. PLOS ONE 2017; 12(11):e0188274. doi: 10.1371/journal.pone.0188274.

36. Hummel M, Edelmann D, Kopp-Schneider A. CluMix R package version 2.3.1. 2019.

37. Yoshida K, Bartel A. Tableone: Create 'Table 1' to Describe Baseline Characteristics with or without Propensity Score Weights. R package version 0.12.0. 2020. URL https://CRAN.Rproject.org/package=tableone.

38. Hastie T. gam: Generalized Additive Models. R package version 1.20. 2020. URL https://CRAN.Rproject.org/package=gam.

\section{Figures}




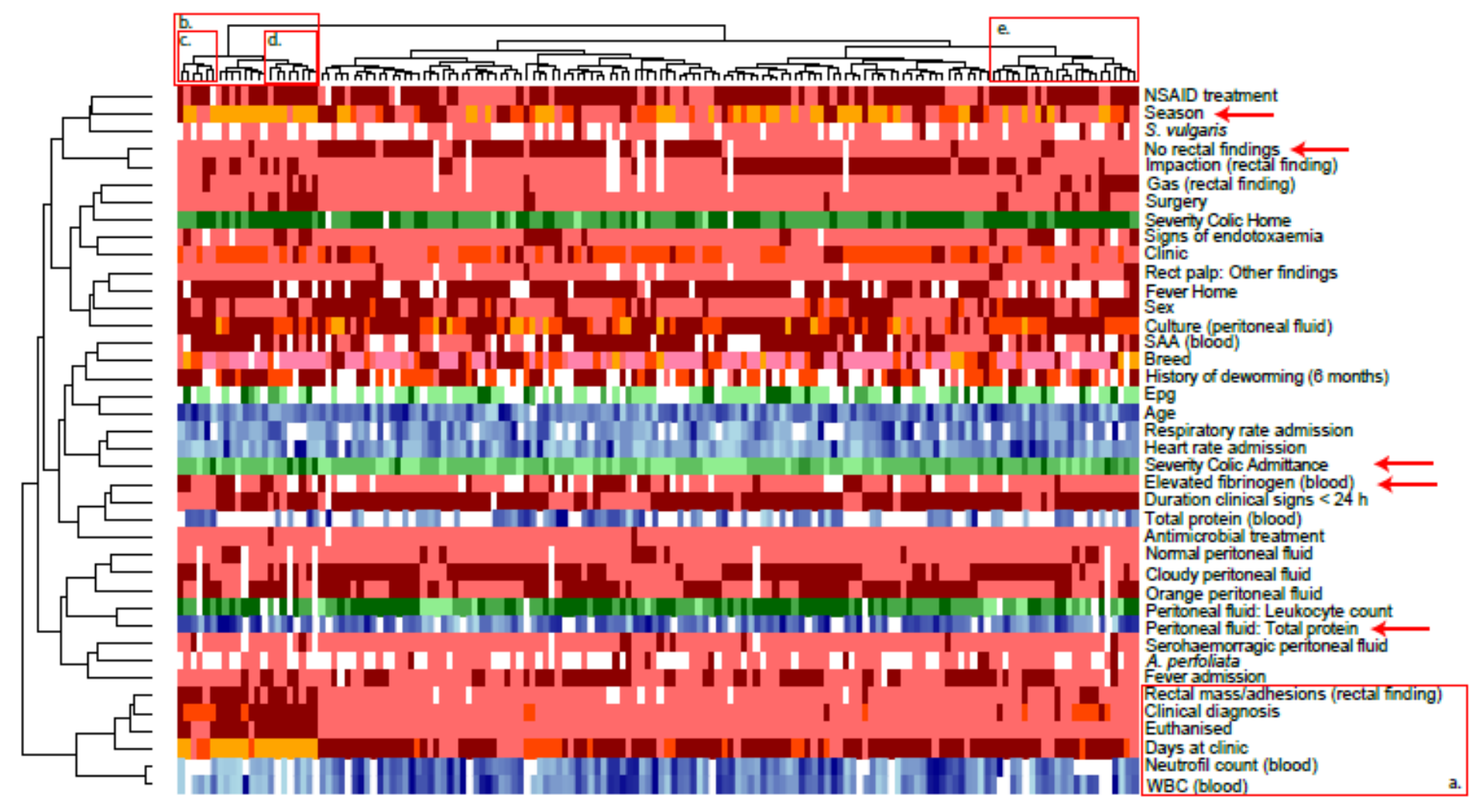

Quantitative vatiabies

Ordinal variabies

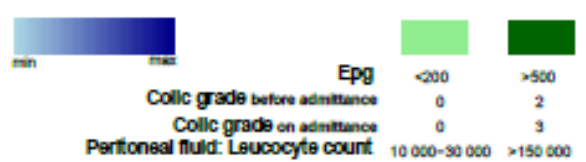

Categorical variebies

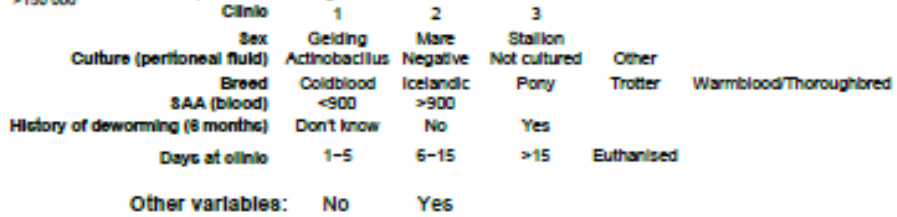

\section{Figure 1}

Cluster analysis. 


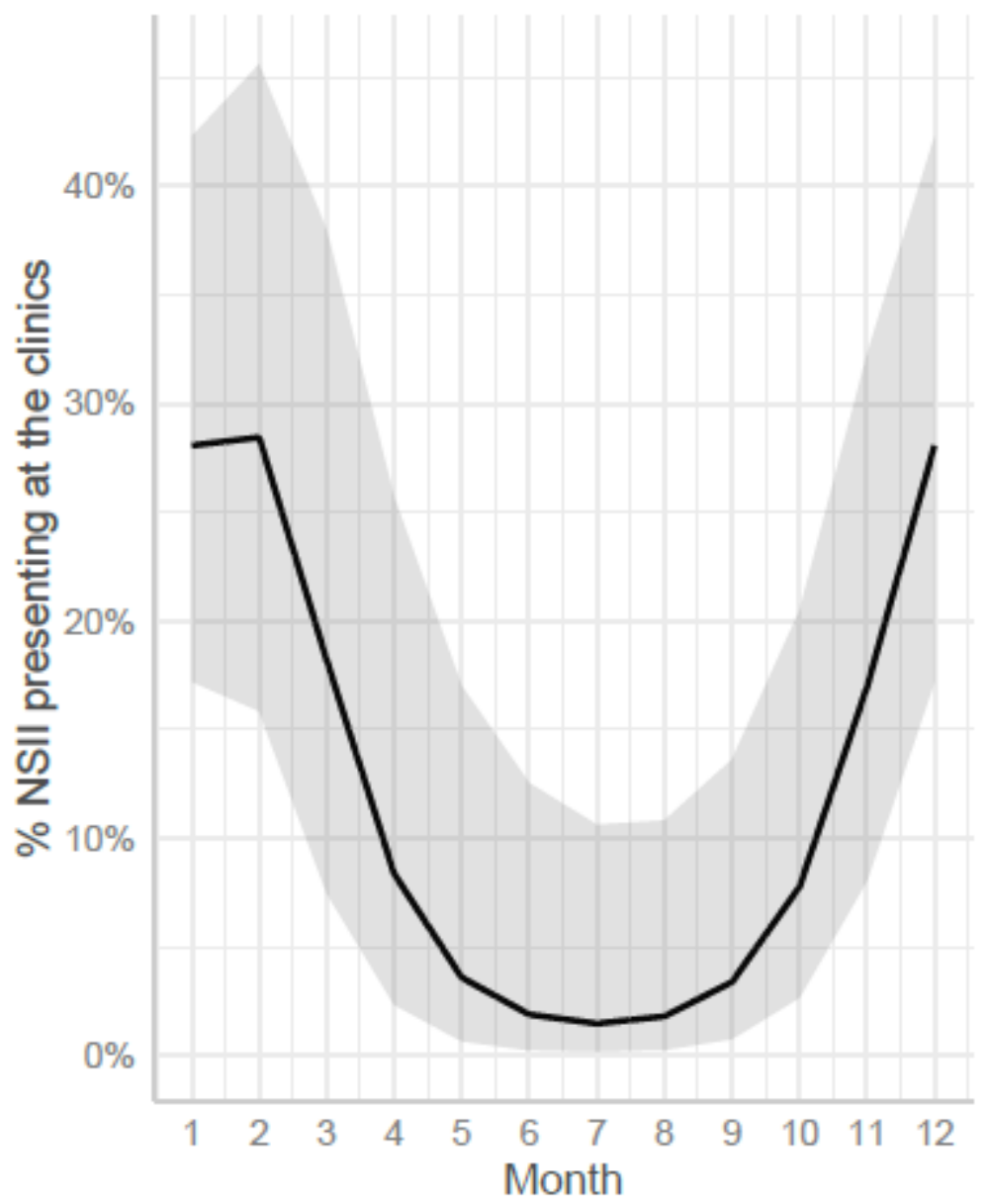

Figure 2

Predicted probabilities of a confirmed NSII diagnosis according to month of presentation.
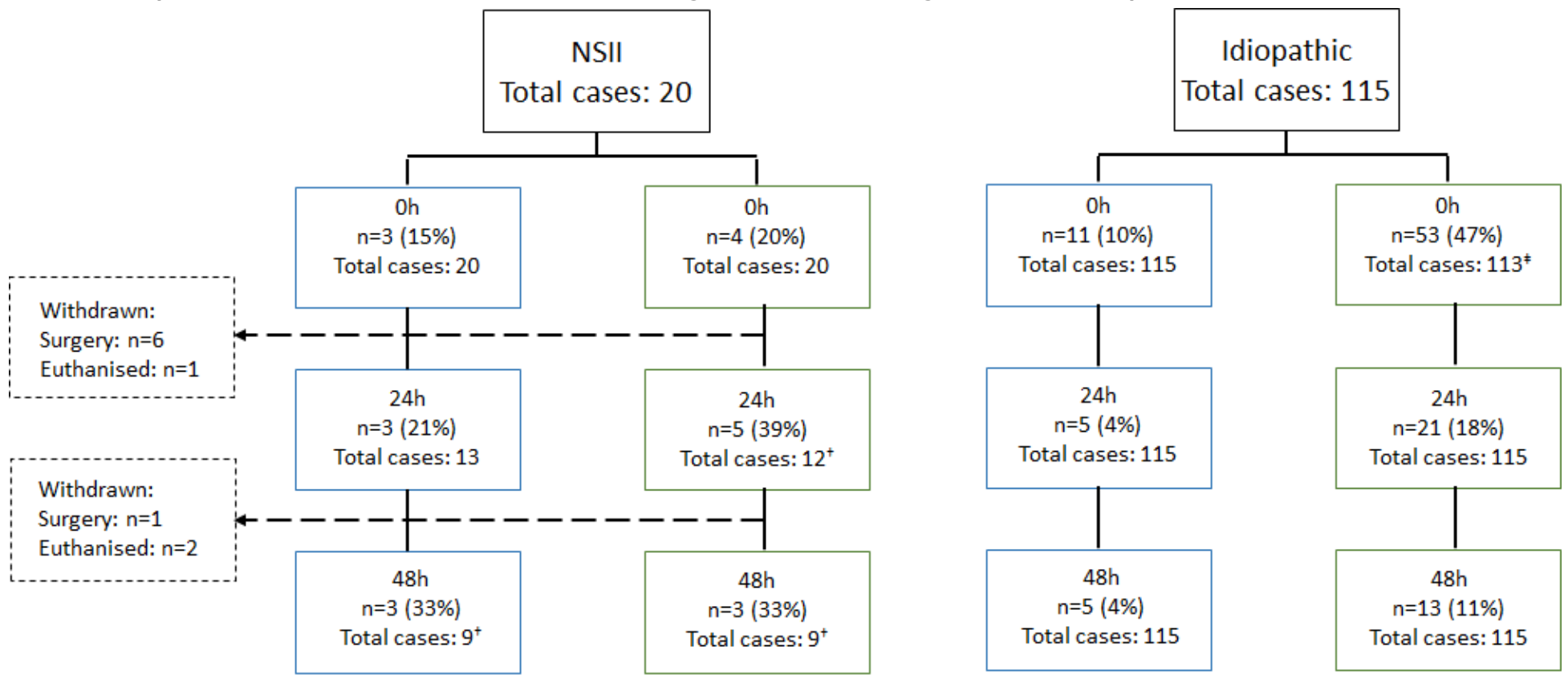

${ }^{\dagger}$ One case missing, ${ }^{\ddagger}$ two cases missing

Blue box $=$ colic signs grade 2 or 3 , green box $=$ fever $\left(\geq 38.5^{\circ} \mathrm{C}\right)$

Page $20 / 23$ 


\section{Figure 3}

Number of cases with colic and fever at admission and after 24 and 48 hours of commencement of medical treatment for each study group (one case in the idiopathic group was not treated). The number of NSII horses decreases with time due to either surgical intervention or euthanasia.
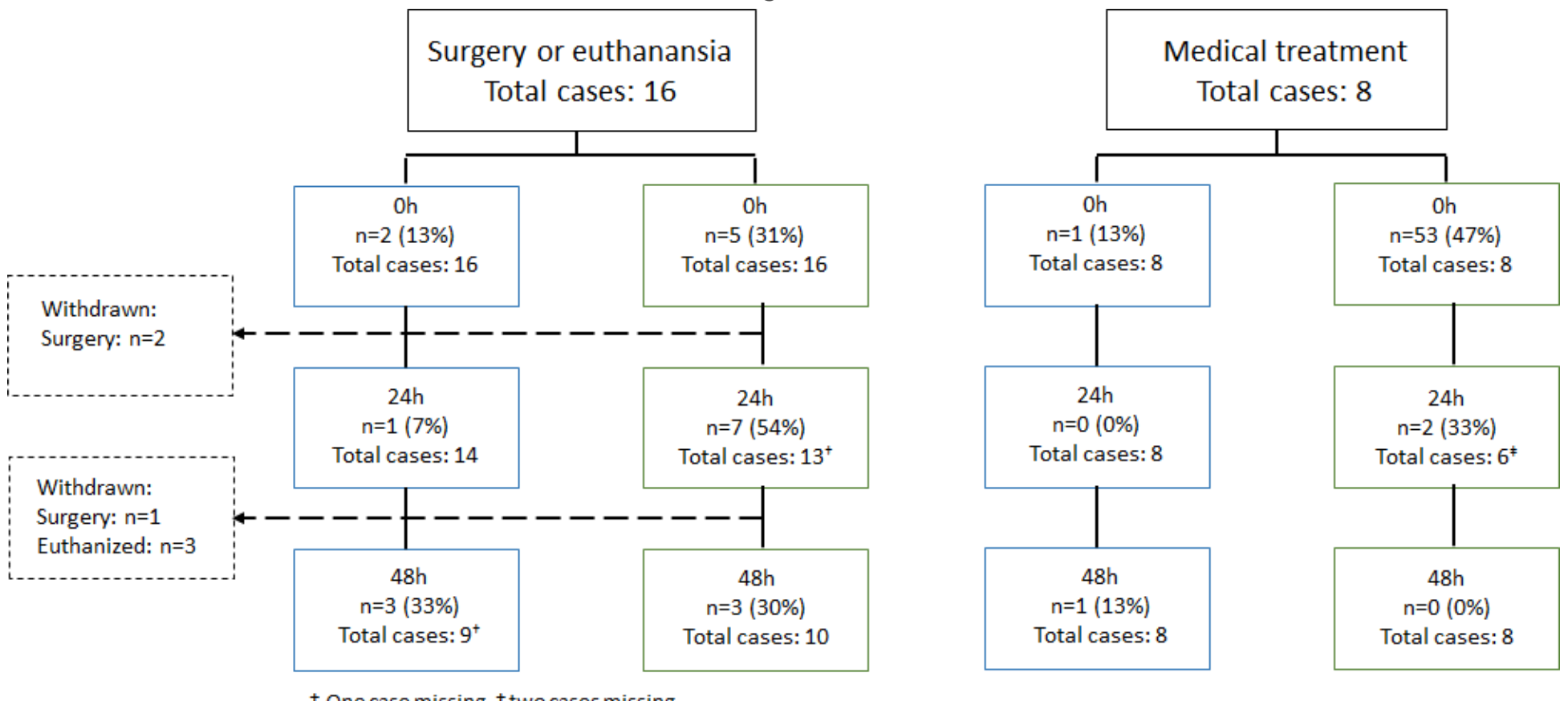

† One case missing, $¥$ two cases missing

Blue box $=$ colic signs grade 2 or 3 , green box $=$ fever $\left(\geq 38.5^{\circ} \mathrm{C}\right)$

\section{Figure 4}

Number of cases with colic and fever at admission and after 24 and 48 hours of commencement of medical treatment in horses with rectal and/or ultrasonographic findings suggestive of NSII. 


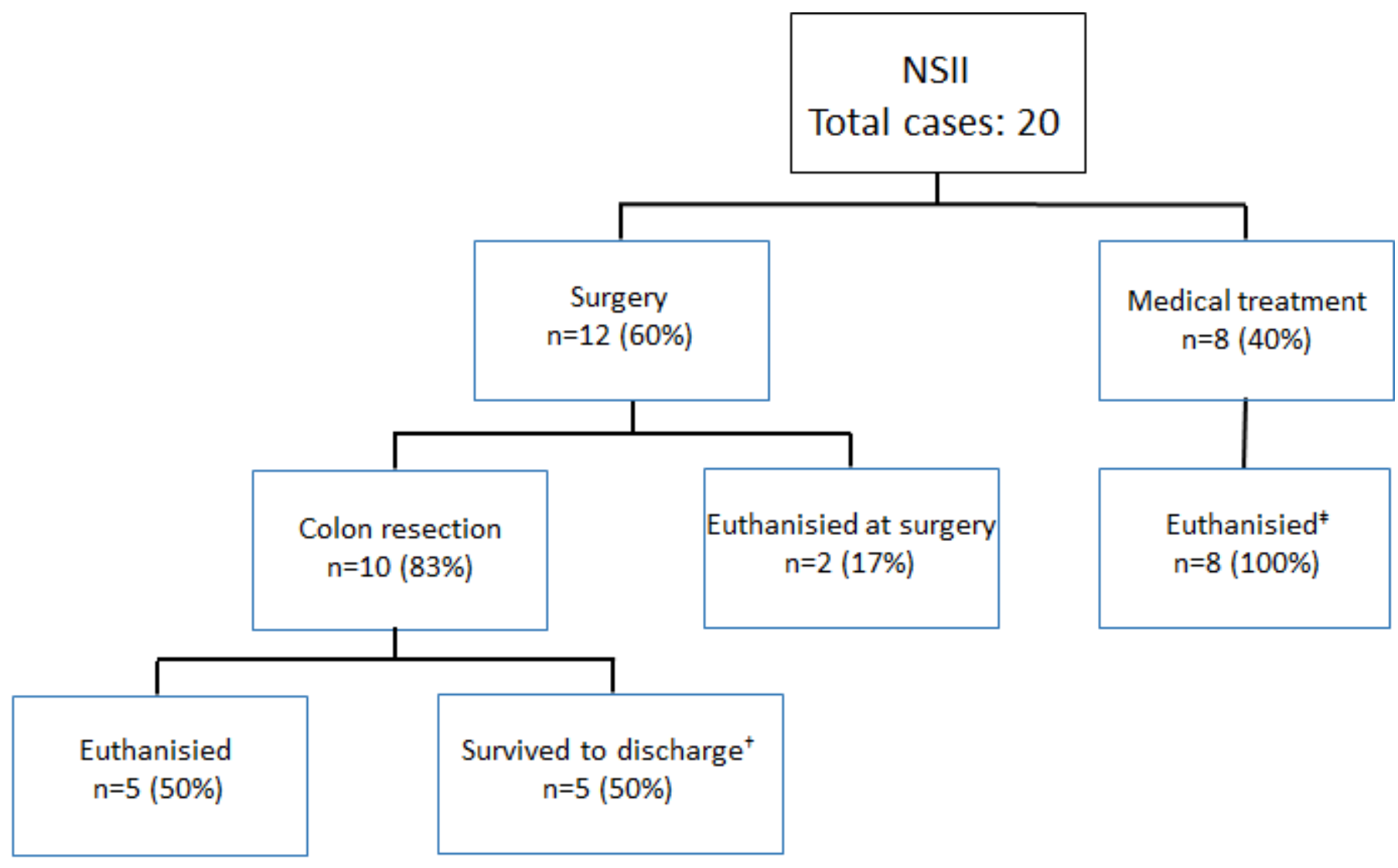

${ }^{+}$Of which one horse was euthanized 9 months after discharge due to stricture formation.

₹Euthanized between 1-4 days from admission.

\section{Figure 5}

Outcome in horses with confirmed NSII.

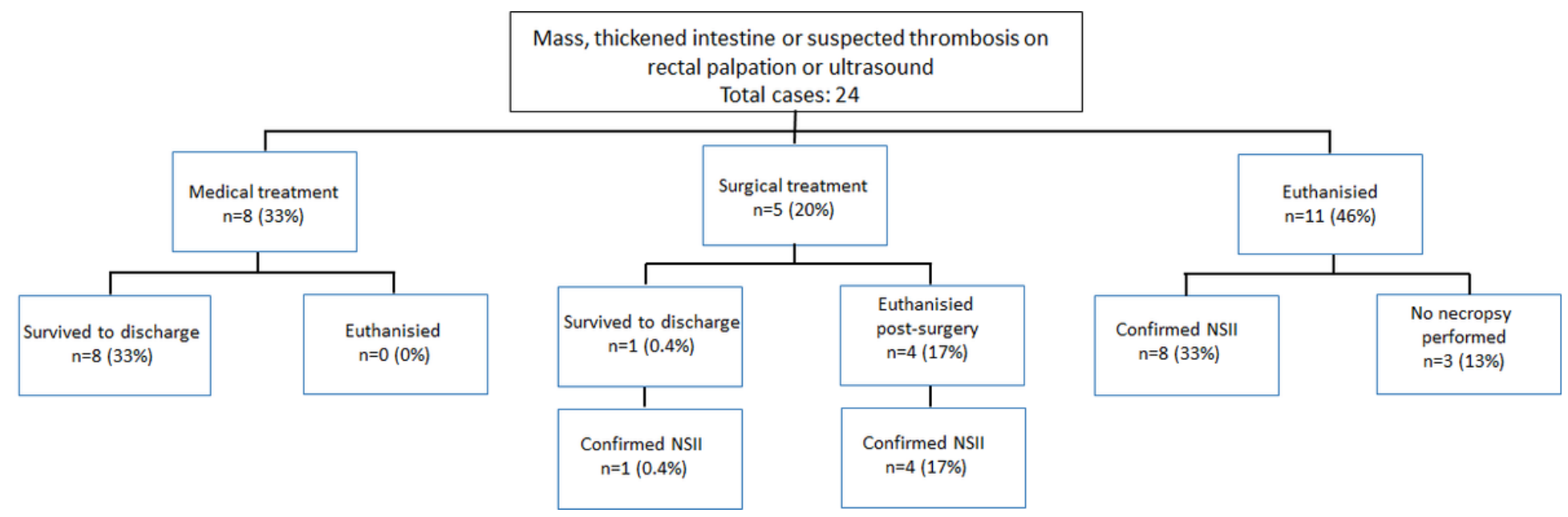

Figure 6

Outcome in horses with rectal and/or ultrasonographic findings suggestive of NSII. 


\section{Figure 7}

A. An acute non-strangulating intestinal infarction in the left ventral colon. B. Adhesion formation between a non-strangulating intestinal infarction in the pelvic flexure and the abdominal wall and displacement of the caecal base with adhesion formation to the left ventral colon, found at necropsy. C. Verminous arteritis of the cranial mesenteric artery with presence of S. vulgaris larvae.

\section{Supplementary Files}

This is a list of supplementary files associated with this preprint. Click to download.

- Suppl.Figure1a..pdf

- Suppl.Figure1b..pdf

- Suppl.Table1a..docx

- Suppl.Table1b..docx

- Suppl.Table1c..docx

- Suppl.Table1d..docx 